\title{
PEAK: Revisão de Literatura das Intervenções Baseadas em Equivalência de Estímulos e RFT para Pessoas com Desenvolvimento Atípico
}

\section{PEAK: Literature Review of the Interventions Based on Stimulus Equivalence and RFT for People with Atypical Development}

\section{PEAK: Revisión de Literatura de las Intervenciones Basadas en Equivalencia de Estímulos y RFT para Personas con Desarrollo Atípico}

\author{
Izaniele Marquetti ${ }^{1}$, Yanne R. Gonçalves ${ }^{2}$, Ana Raquel Q. Amaral ${ }^{3}$
}

[1] Centro Paradigma de Ciências do Comportamento [2] Universidade Federal do Paraná [3] Universidade de Brasília I Título abreviado: PEAK: Equivalência de estímulos e RFT I Endereço para correspondência: Ana Raquel Queiroz Amaral - Departamento de Processos Psicológicos Básicos/lnstituto de Psicologia, Universidade de Brasília, Campus Universitário Darci Ribeiro, Brasília - DF; CEP: 70.910-900 | Email: amaral.anarq@gmail.com I doi: 10.18761/PAC.2021.v12.RFT.16

Resumo: O PEAK é um instrumento de avaliação e intervenção comportamental com proposta inovadora. O objetivo do presente artigo foi investigar as possibilidades do instrumento para intervenção em equivalência e transformação (RFT). A coleta de dados compreendeu o período de 2014 até 2019 e foi realizada inicialmente no site oficial do instrumento e depois ampliada para periódicos da área. As palavras utilizadas na pesquisa foram "PEAK", "equivalência" e "transformação". Os resultados mostraram que o currículo foi eficaz para o ensino de diferentes populações como pessoas com autismo, síndrome de Down e deficiência intelectual. Foram ensinados diferentes conteúdos, como habilidades acadêmicas, relações de sistemas sensoriais e habilidades mais complexas em linguagem e tomada de perspectiva. Como limitações, foram observadas a idade dos participantes e o fato de a maioria dos estudos ter sido realizada pelo grupo de pesquisa do autor do instrumento. Sugerem-se novas investigações.

Palavras-chave: aprendizagem derivada, treino de habilidades, neurodiversidade, transformação.

\begin{abstract}
PEAK is a behavioral assessment and intervention instrument with an innovative proposal. This article aimed to investigate the possibilities of PEAK equivalence and transformation modules (RFT) as a tool of intervention. The timespan of selected data was from 2014 to 2019. The search was initially carried out on the official website of the instrument and later expanded to journals of the area. The search expressions were "PEAK", "equivalence", and "transformation". The results showed that the curriculum was effective for teaching different populations such as people with autism, Down syndrome, and intellectual disability. Different contents were taught, such as academic skills, sensory system relations, and more complex skills in language and perspective. As limitations, it was observed the age of the participants and the fact that the majority of studies was carried out by the research group of the author of the instrument. Further investigations are suggested.
\end{abstract}

Keywords: derived learning, skills training, neurodiversity, transformation 
Resumen: PEAK es una herramienta de evaluación e intervención conductual con una propuesta innovadora. El propósito de este artículo fue investigar las posibilidades del instrumento de intervención en equivalencia y transformación (RFT). La recolección de datos abarcó el período de 2014 a 2019 e inicialmente se llevó a cabo en el sitio web oficial del instrumento y luego se extendió a periódicos del área. Las palabras utilizadas en la investigación fueron "PEAK", "equivalencia" y "transformación". Los resultados mostraron que el plan de estudios fue eficaz para enseñar a diferentes poblaciones, como personas con autismo, síndrome de Down y discapacidad intelectual. Se impartieron diferentes contenidos, como habilidades académicas, relaciones del sistema sensorial y habilidades más complejas en lenguaje y perspectiva. Como limitaciones, observamos la edad de los participantes y la constatación de la mayoría de los estudios haber sido realizada por el grupo de investigación del autor del instrumento. Se sugieren más investigaciones.

Palabras clave: aprendizaje derivado, entrenamiento de habilidades, neurodiversidad, transformación.

Agradecimento especial a nossa colaboradora, Dra. Mayara Juliana Pares, que ajudou a encontrar os artigos, leu nosso texto e fez correções - e, finalmente, auxiliou na formatação do artigo. Somos gratas pela sua contribuição.

Agradecemos também à mestranda Fernanda Cristina Ribeiro Nisihara, que prontamente aceitou catalogar os artigos para a realização do acordo entre observadores. 
O PEAK - Promoting the Emergence of Advanced Knowledge - Relational Training System refere-se à avaliação e ao currículo elaborados por Mark R. Dixon, com a publicação do primeiro livro em 2014 (Dixon, 2014a). Sua proposta é promover habilidades cognitivas e de linguagem, usando abordagens de comportamento verbal, em conjunto com os avanços das pesquisas em equivalência de estímulos (Sidman, 1971) e da RFT - teoria das molduras relacionais (Hayes et al., 2001), aplicada a indivíduos com atrasos no desenvolvimento (Belisle, Dixon, \& Stanley, 2018).

A teoria do comportamento verbal proposta por Skinner (1957/1978) em seu livro Verbal Behavior tem sido largamente utilizada nas últimas décadas no estudo, avaliação e intervenção relativos ao desenvolvimento da linguagem, principalmente de pessoas com desenvolvimento atípico (Dixon et al., 2007). A proposta de Skinner (1957/1978) é que o desenvolvimento da linguagem humana acontece de forma operante, por meio de uma história de reforçamento direto, mediado por um ouvinte especialmente treinado. Diversos instrumentos avaliativos foram elaborados com base da obra de Skinner (1957/1978), embasados especialmente nas categorias criadas por ele, denominadas operantes verbais (e.g., tato, mando, autoclítico, etc.), como, por exemplo, o VBMAPP - Verbal Behavior Milestones Assessment and Placement Program e o ABLLS-R - The Assessment of Basic Language and Learning Skills - Revised (Dixon et al., 2017).

No entanto, a dependência da exposição a contingências de reforçamento e punição para o desenvolvimento da linguagem é o cerne das críticas levantadas contra a teoria de Skinner (Hayes et al., 2001). Uma crítica famosa foi realizada pelo linguista Chomsky (1956), segundo o qual a teoria skinneriana não daria conta de explicar a geratividade da linguagem.

Nesse sentido, avanços relacionados à equivalência de estímulos e à RFT estão cada vez mais apoiando uma explicação comportamental que considere prontamente a complexidade e a emergência de comportamento novos, relacionada ao desenvolvimento da linguagem e cognição humana (Dymond et al., 2010).

Diferentemente das avaliações exclusivamente baseadas nos operantes verbais, o PEAK incor- pora distintas faces da literatura analítico-comportamental, como: tentativa discreta (fracionar o objetivo em partes e apresentá-las uma de cada vez, reforçando as respostas corretas e corrigindo as incorretas); equivalência de estímulos; e a RFT (Dixon, Belisle, et al., 2014). Esse instrumento foi construído mapeando o repertório de pessoas com habilidades até 16 anos de idade, movido também pelo aumento da demanda para intervenção em análise do comportamento aplicada com pessoas com autismo (Dixon, Wiggins, \& Belisle, 2018).

O PEAK é composto por quatro livros: treino direto (DT), generalização $(\mathrm{G})$, equivalência (E) e transformação $(\mathrm{T})$. Cada livro oferece 184 programas individuais, de forma que, para cada um deles, os profissionais recebem instruções sobre como realizar as avaliações iniciais e, com base nas habilidades atuais, colocar o aprendiz em uma faixa de habilidades apropriada (Dixon, Belisle, Munoz, et al., 2017). Os dois primeiros livros - treino direto e generalização - são similares ao VB-MAPP e a ABLLS-R com relação ao arcabouço teórico (comportamento verbal de Skinner), mas apresentam topografias de operantes verbais mais complexas em comparação aos instrumentos citados (Dixon, Belisle, Munoz, et al., 2017).

O primeiro livro, especificamente, enfatiza a formação da linguagem e habilidades de aprendizagem por meio de ensino a partir de tentativa discreta, ou seja, fornecendo reforço para respostas corretas e correção para respostas incorretas (Dixon, Belisle, et al., 2014). Nesse livro, são avaliadas as habilidades que o autor denomina aprendizagens fundamentais, aprendizagem perceptiva, compreensão verbal, raciocínio verbal, memória e matemática (Dixon, Belisle, Munoz, et al., 2017). As habilidades são apresentadas em um nível crescente de dificuldade, desde habilidades muito básicas, como contato visual e imitação motora, até mais complexas, como contar piadas e uso de linguagem metafórica (McKeel, Rowsey, Belisle, et al., 2015). Em termos de validade, observa-se forte correlação com testes de inteligência (Belisle, Dixon, \& Stanley, 2018), teste de vocabulário de figuras e VBMAPP (Dixon, Belisle, Stanley, \& Rowsey, 2016).

No segundo livro, generalização, a proposta é que o indivíduo já tenha passado pelo treino direto e desenvolva um repertório amplo (Dixon, 2014b). 
A ideia é que a criança consiga aplicar as habilidades aprendidas em outras situações, sendo capaz de utilizar o repertório desenvolvido nas terapias em situações similares da sua vida diária. É devido à generalização que os indivíduos aprendem novos comportamentos sem a necessidade de modelagem de uma mesma resposta em vários contextos diferentes (Matsumoto, 2014). Nesse livro, são abarcadas aprendizagem fundamental e habilidades sociais básicas; compreensão verbal básica, memória e habilidades sociais avançadas; compreensão verbal avançada, resolução de problemas básica e habilidades matemáticas avançadas; raciocínio verbal, resolução de problemas avançada e habilidades de leitura e escrita avançadas (Dixon, Belisle, Munoz, et al., 2017).

Já no terceiro livro do PEAK (equivalência) são inseridos os conceitos relativos a equivalência de estímulos, que complementam as avaliações habituais (Dixon, 2015). O foco em uma abordagem baseada na equivalência de estímulos é no ensino de relações (condicionais) arbitrárias entre estímulos e na emergência de novas respostas que não são diretamente reforçadas, mas que derivam das relações inicialmente treinadas (Sidman \& Tailby, 1982). Quando isso acontece, observa-se que os estímulos relacionados durante o treino são substituíveis entre si em determinados contextos - e, portanto, equivalentes; por exemplo, a palavra falada "bola" (A), a palavra escrita "BOLA" (B) e a própria bola (objeto - C) da qual a palavra falada e a palavra escrita fazem referência tornam-se substituíveis dependendo do contexto em que são utilizadas. Tais estímulos formam uma classe de equivalência na medida em que tais relações de substitutibilidade apresentem as propriedades de reflexividade (e.g., $A=A$ ), simetria (e.g., se $A=B$, emerge sem treino direto a relação $\mathrm{B}=\mathrm{A}$ ) e transitividade (e.g., após o treino de $\mathrm{A}=\mathrm{B}$ e $\mathrm{B}=\mathrm{C}$, emerge sem treino a relação $\mathrm{A}=\mathrm{C})$ (McIlvane, 2014; Sidman \& Tailby, 1982). O PEAK avalia as três propriedades definidoras da formação de classes de equivalência (Dixon, Belisle, Munoz, et al., 2017).

Por fim, o último livro, transformação, é baseado na RFT (Dixon, 2016). Segundo Hayes et al. (2001), o responder relacional arbitrariamente aplicado (RRAA) consiste nas respostas que não foram diretamente reforçadas e são observadas de forma previsível. O RRAA é estabelecido por meio de uma história específica de treino de múltiplos exemplares e depende do controle contextual (Hayes et. al., 2001; Torneke, 2010).

Além da possibilidade de estudar e treinar esse repertório complexo, uma das grandes vantagens do uso da RFT é a economia de treino, visto que, ao se treinar algumas relações, outras tantas derivam sem treino direto, assim como na equivalência de estímulos. No entanto, aqui se compreende que a aprendizagem vai além de relacionar estímulos enquanto iguais. Cada tipo de relação foi denominada moldura relacional, e são elas: coordenação (relacionar iguais); distinção (relacionar diferentes); oposição (relacionar opostos); comparação (comparar estímulos); hierarquia (relacionar enquanto classe ou categoria); espacial (relacionar localizações); temporal (relacionar questões de temporalidade) e relações dêiticas (relações complexas de tomada de perspectiva) (Hayes et. al., 2001; Torneke, 2010).

Na RFT, os RRAAs são definidos por três propriedades: (1) implicação mútua: dado um estímulo A relacionado com $\mathrm{B}$, logo $\mathrm{B}$ está relacionado com A; (2) implicação mútua combinatória: dado um estímulo A relacionado com B e B relacionado com C, $\log o$ B está relacionado com C; e (3) transformação de função: função de um estímulo é transformada a depender do tipo de relação implicada com outro(s) estímulo(s). Por meio da transformação de função, estímulos podem adquirir funções, as mais diversas, indiretamente: funções discriminativas, reforçadoras, punitivas ou eliciadoras (Dymond \& Rehfeldt, 2000; Hayes et. al., 2001; Perez et al., 2013).

A partir do exposto, entende-se que a equivalência de estímulos e a teoria das molduras relacionais são explicações teóricas fundamentais para o entendimento do desenvolvimento, que complementam as ideias trazidas por Skinner (1957/1978). Nesse cenário, o PEAK surge com a proposta de reunir, em um único instrumento, as atualizações teóricas e científicas da análise do comportamento aplicada, ou ABA (do inglês applied behavior analysis) para o estudo e ensino dessas habilidades que não são abarcadas em avaliações e currículos já amplamente utilizados em intervenções com pessoas com atraso no desenvolvimento. Com base nesses diferenciais, faz-se importante um estudo 
para apresentar tal instrumento, seus diferenciais e possibilidades de atuação em intervenções baseadas em ABA.

Em 2017, foi publicada uma revisão de literatura incluindo artigos de 2014 a 2017, realizada pelo criador do instrumento, Marx Dixon, e colaboradores (Dixon, Belisle, McKeel, et al., 2017). Nessa revisão, os autores analisaram a validade social, dados demográficos dos estudos, avaliações psicométricas e eficácia do treinamento a partir de delineamentos entre grupos e de sujeito único, assim como as limitações de cada pesquisa.

No que diz respeito às propriedades psicométricas, os autores concluíram que os dois primeiros livros, treinamento direto e generalização, apresentaram boa confiabilidade, e os dois últimos não foram analisados. Em relação à validade, o PEAK demonstrou forte correlação com outros instrumentos avaliativos já consolidados, também com foco nos dois primeiros livros. No que diz respeito à eficácia do tratamento, foi apresentado apenas um estudo com delineamento de grupo com diferença significativa no pré e pós-teste, fato não observado no grupo-controle. O PEAK também foi eficaz nos estudos de intrassujeitos para o ensino de uma ampla gama de alvos verbais e acadêmicos avançados.

O presente artigo tem por objetivo atualizar a revisão de literatura realizada por Dixon, Belisle, McKeel, et al. (2017), incluindo também os estudos realizados entre 2017 e 2019. Ressaltamos que esse é, dentro do nosso conhecimento, possivelmente a primeira revisão realizada por um grupo de pesquisadores independente, sem vínculo com os proponentes do instrumento. Além disso, especificamente, tem-se por objetivo avaliar os estudos referentes aos dois últimos livros, equivalência $\mathrm{e}$ transformação, livros estes focados na avaliação e desenvolvimento das características gerativas do repertório verbal.

\section{Método}

A pesquisa de revisão foi dividida em duas partes. Inicialmente, referências foram buscadas no próprio site do PEAK (https://www.peak2aba.com) que conta com uma lista dos artigos publicados sobre o tema do grupo criador do instrumento. $\mathrm{Na}$ sequência, foram selecionadas todos os periódicos que tinham as referidas publicações. Foram encontrados 13 periódicos, a saber: Behavior Analysis in Practice, Journal of Applied Behavior Analysis, Journal of Organizational Behavior Management, Journal of Contextual Behavioral Science, The Behavior Analyst, Behavior Analysis: Research and Practice, The Psychological Record, Behavior Analysis and Therapy, Journal of Developmental and Physical Disabilities, The Analysis of Verbal Behavior, Journal of Behavioral Education, Behavior Analysis International, Behavior Analysis in Practice e Research in Autism Spectrum Disorders.

Posteriormente, no site de cada periódico, foi realizada uma busca com as palavras-chave "PEAK", "equivalência" e "transformação". Foram selecionadas todas as publicações sobre o PEAK, entre os anos de 2014 e 2019. Nessa etapa, foram localizados quatro estudos que não estavam listados no site do PEAK: Dixon, Carman, et al. (2014); Reed e Luiselli (2016); Witts (2018); e Daar et al. (2015).

Ao todo, foram encontrados 39 artigos. Os artigos foram lidos parcialmente por duas autoras (resumo e método). Foram selecionados para análise artigos que se incluíssem nos seguintes critérios: (1) que se referissem aos livros de equivalência e transformação e (2) que apresentassem resultados da aplicação de pelo menos um desses livros com ao menos um participante com atraso no desenvolvimento. Dos 39 artigos selecionados inicialmente, 13 enquadraram-se nos critérios de análise e foram incluídos na tabulação dos dados, nas categorias: revista, título, autor, ano, livro do PEAK, participantes, delineamento, resultados e limitações.

Um observador independente realizou a leitura de cinco dos 13 artigos e a tabulação nas categorias de análise. Os dados de comparação foram avaliados seguindo um método de comparação ponto a ponto: o número de concordâncias foi dividido pelo número total de comparações e depois multiplicados por 100. O índice de acordo entre observadores foi de $85 \%$. 


\section{Resultados}

A Tabela 1 sintetiza as informações extraídas dos 13 artigos. Foram encontrados oito estudos que utilizaram o livro de equivalência, quatro que empregaram o livro de transformação e um que utilizou ambos.

No total, os artigos contemplaram 80 participantes entre crianças, adolescentes e adultos. Todos tinham alterações no desenvolvimento, sendo 71 diagnosticados com autismo, dois com síndrome de Down, quatro com deficit intelectual e três com atraso no desenvolvimento sem especificações.

Em relação ao delineamento utilizado nos estudos, todos usaram delineamento intrassujeito, com exceção de Belisle et al. (2017), que realizou um estudo de grupo e correlacionou o desempenho nas habilidades de equivalência do PEAK com o resultado do instrumento de avaliação funcional QABF.

Tabela 1. Descrição das Principais Características dos Estudos Revisados

\begin{tabular}{|c|c|c|c|c|c|}
\hline \multicolumn{6}{|c|}{ Livro: Equivalência } \\
\hline Id. & Título & Autores/Ano & Participantes/Design & Resultados & Limitações \\
\hline 1 & $\begin{array}{l}\text { A relação entre as } \\
\text { relações derivadas } \\
\text { mutuamente implicadas } \\
\text { e a função do compor- } \\
\text { tamento desafiador em } \\
\text { crianças com autismo: } \\
\text { Comparando PEAK-E- } \\
\text { PA e QABF. }\end{array}$ & $\begin{array}{l}\text { Belisle et al. } \\
(2017)\end{array}$ & $\begin{array}{l}47 \text { indivíduos sendo } \\
43 \text { com autismo, } \\
2 \text { com síndrome } \\
\text { de Down e } 2 \text { com } \\
\text { Deficiência Intelectual/ } \\
\text { Correlação entre } \\
\text { as duas avaliações } \\
\text { (PEAK e QABF). }\end{array}$ & $\begin{array}{l}\text { - As pontuações gerais no } \\
\text { QABF eram menores para } \\
\text { os participantes capazes de } \\
\text { derivar relações mutuamente ou } \\
\text { combinatoriamente implicadas. } \\
\text { - Para indivíduos capazes de } \\
\text { derivar estas relações o QABF } \\
\text { não conseguiu isolar uma única } \\
\text { função do comportamento } \\
\text { avaliado } \\
\text { - A capacidade de derivar } \\
\text { relações não foi preditiva de } \\
\text { nenhuma topografia específica } \\
\text { e se relacionava com as pon- } \\
\text { tuações do QABF somente na } \\
\text { subescala física. }\end{array}$ & $\begin{array}{l}\text { - Amostra pequena. } \\
\text { - Comportamentos desafiadores } \\
\text { foram selecionados por avaliação } \\
\text { indireta feita pelos professores de } \\
\text { forma retrospectiva, sem garantia } \\
\text { de que eram os mais frequentes } \\
\text { ou mais válidos socialmente para } \\
\text { estratégias de redução de compor- } \\
\text { tamento. } \\
\text { - Diferença no número de partici- } \\
\text { pantes entre os dois grupos. } \\
\text { - Apesar de terem sido encontradas } \\
\text { diferenças significativas entre os } \\
\text { grupos no uso do QABF para isolar } \\
\text { a função do comportamento, outro } \\
\text { resultado poderia ter sido encontra- } \\
\text { do se o critério estabelecido fosse } \\
\text { diferente. } \\
\text { - Não foram realizados esforços } \\
\text { para controlar a gravidade de outros } \\
\text { sintomas associados ao quadro de } \\
\text { autismo (p.e. déficits na linguagem). }\end{array}$ \\
\hline 2 & $\begin{array}{l}\text { Avaliando as relações } \\
\text { de nomear emergentes } \\
\text { através de desenho } \\
\text { representacional em in- } \\
\text { divíduos com distúrbios } \\
\text { do desenvolvimento } \\
\text { usando o currículo } \\
\text { PEAK. }\end{array}$ & $\begin{array}{l}\text { Dixon, Belisle, } \\
\text { Rowsey, et al. } \\
\text { (2016) }\end{array}$ & $\begin{array}{l}1 \text { criança com autis- } \\
\text { mo, uma criança com } \\
\text { autismo e TDAH, } 1 \\
\text { criança com atraso } \\
\text { cognitivo e emocional/ } \\
\text { Linha de base múltipla } \\
\text { entre participantes. }\end{array}$ & $\begin{array}{l}\text { - Domínio da relação entre a } \\
\text { figura misturada do animal }(A) \\
\text { e o nome vocal do animal }(B) \\
\text { diretamente reforçado. } \\
\text { - Domínio da relação derivada } \\
\text { entre o nome vocal do animal } \\
\text { (B) e a figura misturada do } \\
\text { animal (A) não treinada. }\end{array}$ & $\begin{array}{l}\text { - Replicação não pode ser realizada } \\
\text { com participantes que ainda não } \\
\text { possam nomear e desenhar. } \\
\text { - Muitos aspectos da nomeação não } \\
\text { foram testados. }\end{array}$ \\
\hline 3 & $\begin{array}{l}\text { Relações de equiva- } \\
\text { lência derivadas de } \\
\text { habilidades de geome- } \\
\text { tria em estudantes com } \\
\text { autismo: uma aplicação } \\
\text { do currículo PEAK-E. }\end{array}$ & $\begin{array}{l}\text { Dixon, Belisle, } \\
\text { Stanley, Daar, } \\
\& \text { Williams } \\
(2016)\end{array}$ & $\begin{array}{l}2 \text { adolescentes com } \\
\text { autismo/ Linha de } \\
\text { base múltipla entre } \\
\text { participantes. }\end{array}$ & $\begin{array}{l}\text { - Domínio da relação direta- } \\
\text { mente reforçada vocal nome da } \\
\text { forma (A)- Vocal propriedade } \\
\text { da forma (B) } \\
\text { - Domínio da relação derivada } \\
\text { não treinada Vocal propriedade } \\
\text { da forma (B) - Vocal nome da } \\
\text { forma (A) } \\
\text { - Domínio da relação derivada } \\
\text { transitiva não treinada Vocal } \\
\text { nome da forma (A)- figura da } \\
\text { forma (C) }\end{array}$ & $\begin{array}{l}\text { - Participantes tinham boas habilida- } \\
\text { des relacionais antes do estudo; } \\
\text { - As habilidades de identificar "la- } \\
\text { dos" das formas não foram testadas } \\
\text { antes do estudo; } \\
\text { - Não foram conduzidas provas } \\
\text { testando B-A ou A-C durante a fase } \\
\text { de treinamento. }\end{array}$ \\
\hline
\end{tabular}


$4 \quad$ Estabelecimento de classes de equivalência auditivo-tátil-visual em crianças com autismo e atraso no desenvolvimento.

$\begin{array}{ll}\text { Mullen et al. } & 2 \text { crianças com } \\ \text { (2017) } & \text { autismo/ Linha de } \\ \text { base múltipla entre } \\ \text { participantes. }\end{array}$

Stanley et al. (2018) equivalência de habilidades acadêmicas: aplicação em adolescentes com autismo.

$6 \quad$ Utilizando o sistema de treinamento relacional PEAK para ensinar relações visuais, gustativas e auditivas a adultos com deficiências de desenvolvimento.

3 adolescentes autismo/ Linha de base múltipla entre participantes com múltiplas sondas.

$\begin{array}{ll}\text { McKeel \& } & 3 \text { participantes com } \\ \text { Matas } & \text { autismo adultos/ } \\ \text { (2017b) } & \text { Duas linhas de base } \\ & \text { múltiplas entre partici- } \\ & \text { pantes com múltiplas } \\ & \text { sondas. }\end{array}$
diagnosticados com

Os dois participantes obtiveram avanços, incluindo sondagens nunca treinadas.

A: estímulos gustativos.

B: figuras dos sabores.

C: estímulo vocal de cada sabor.

Sondagens demonstram que após treino B-C os participantes foram capazes de realizar a simetria C-B.

Um dos participantes foi capaz de realizar transitividade.

Eficácia em utilizar equivalência de estímulos para ensinar habilidades acadêmicas avançadas. Promoveu aprendizado de habilidades generalizadas.

Todos os participantes obtiveram critério durante $o$ treinamento e foram capazes de aprender novas habilidades sem treino direto.

A-B: gustativo e figuras.

B-C: figura e auditivo.

Um dos participantes levou mais sessões para obter critério B-C.

Sondagens A-B e B-C mostram que os resultados ocorrem somente com treino.

Domínio para critérios A-C.

\section{$7 \quad$ Estabelecendo} relações de equivalência derivadas de habilidades básicas de geografia em crianças com autismo.

$\begin{array}{ll}\text { Dixon, Stanley, } & 2 \text { crianças com au- } \\ \text { et al. (2017) } & \text { tismo/ } \\ & \text { Linha de base múltipla } \\ & \text { entre participantes. }\end{array}$

Treino de equivalência de estímulos foi relevante para ensino de habilidades acadêmicas com pessoas com TEA, bem como promoveu aprendizado de habilidades não treinadas.
A: nome
B: mapa
C: bandeira
D: continente
Lily respondeu com $100 \%$ de acurácia nos treinos B-C, C-D-, D-A e D-B.
Parker respondeu com $100 \%$ de acuraria nos treinos, com exce- ção do C-A e C-D que foi $80 \%$.

Pequena porcentagem de sessões. Por conta dos estímulos arbitrários usados, a utilidade dos alvos e habilidades treinadas pode ser limitada.

Tentativas aplicadas não foram em mesmo número para todos os participantes. A aplicação ocorreu por pessoas experientes, indicando possíveis dificuldades para implementação por pessoas não treinadas. Omissão dos testes de simetria.

Sondagem de transitividade não foi incluída nas três primeiras sondagens realizadas.

Como foi usado estímulos gustativos, alguns participantes podem não gostar de provar algum item.

Procedimento foi realizado somente por pessoas especialistas. Necessário validar relevância social de professores e cuidadores para aplicação dos procedimentos. 
8 Derivando relações simetricamente coordenadas e gustativas-visuais-auditivas transitivas em crianças com autismo.
Dixon, Belisle, 2 crianças com auStanley, et al. tismo, 1 criança com (2017) atraso cognitivo/ Linha de base múltipla entre habilidades, replicação com 3 participantes.
Domínio da relação diretamente reforçada gustativa $(A)$ - visual (B)

Domínio da relação diretamente reforçada visual (B) - Auditiva (C)

Domínio da relação derivada não treinada simétrica auditiva (C) - visual (B)

Domínio da relação derivada transitiva não treinada gustativa (A) auditiva (C) para 2 dos 3 participantes

Aparecimento de mandos em 2 dos 3.

\section{Livro: Transformação}

9 Ensinando as relações "Antes- Depois" e "Aqui- Lá" para crianças com autismo: Uma avaliação sobre reversões e transformação da função de estímulos.

10 Ensinando as habilidades de tomada de perspectiva fundamentais para crianças com autismo usando o currículo de PEAK-T: reversão única quadros dêiticos "eu- você".

11 Ensinando crianças com autismo a identificar eventos privados de outras pessoas em contexto.

12 Seguimento de regras e transformações derivadas da função de estímulo em um jogo infantil: aplicação em PEAK-E com crianças com deficiências de desenvolvimento.

\section{Barron et al. 2 crianças com autis-} (2019) mo/linha de base múltipla entre habilidades.

Belisle, Dixon, 3 crianças com Stanley, et al. autismo/ linha de (2016) base múltipla com participantes com "sondagem múltipla incorporada".
- Domínio das relações "antes e depois" e "aqui e lá", com transferência da função do estímulo.

- Domínio de reversão para ambas as relações.

- Domínio de transformação para ambas as relações.

- Desenvolvimento da habilidade de tomada de perspectiva. - Para alguns participantes as relações bidirecionais de reversão derivaram do treino de uma relação.

- Desenvolvimentos de transferência da função dos estímulos quando ambas as relações foram treinadas.

\section{Schmick et al. 3 crianças com} (2018) autismo/ Linha de base múltipla entre participantes.

\section{Dixon,} Speelman, et al. (2016)

\section{3 estudantes do sexo} masculino com atraso no desenvolvimento/ Linha de base múltipla entre participantes. para tarefas envolvendo trans-

\section{Facilitou a resposta correta} formação. Foi útil para adolescentes com TEA pois todos representaram situações que poderiam ocorrer na vida real.

$100 \%$ de acertos no jogo Twister com todos os participantes. Todos demonstraram 100\% de acurácia com A-B, B-C regras.

Todos foram capazes de responder corretamente quando foi apresentado um sinônimo no lugar da palavra anatômica.
- Ambos os participantes verbais de alto funcionamento, não representam todos os indivíduos com TEA. - Utilizados somente estímulos visuais. de base para 1 participante. Treinamento misto foi necessário para 1 participante Domínio da relação transitiva não foi demonstrada para 1 participante.
- Utilizada somente a tomada de perspectiva visual.

- Para um dos participantes o resultado para o "eu" foi alto na linha de base e foi necessário treino misto para que dominasse ambas as relações.
Todos tinham repertório verbal.

Não teve generalização para novos estímulos, contextos e emoções. Não foi replicado para outros participantes ou alterada magnitude dos reforçadores durante o treino.

Só 3 classes de estímulos foram apresentadas.

A combinação entre $A-C, C-A$ não foi testada diretamente. Sequência pode ter afetado parte do design.

\section{Livro: Equivalência e Transformação}

\begin{tabular}{|c|c|c|c|c|c|}
\hline 13 & $\begin{array}{l}\text { Emergência derivada } \\
\text { de perguntas e respos- } \\
\text { tas "WH" em crianças } \\
\text { com autismo. }\end{array}$ & $\begin{array}{l}\text { Daar et al. } \\
\text { (2015) }\end{array}$ & $\begin{array}{l}3 \text { crianças com au- } \\
\text { tismo/ } \\
\text { Linha de base múltipla } \\
\text { entre participantes. }\end{array}$ & $\begin{array}{l}\text { Dois dos três participantes } \\
\text { desenvolveram a habilidade } \\
\text { de responder perguntas "WH" } \\
\text { com acurácia. }\end{array}$ & $\begin{array}{l}\text { - Um dos participantes não desen- } \\
\text { volveu a habilidade esperada. } \\
\text { - Só foram avaliadas perguntas } \\
\text { "WH" relacionadas com os estímulos } \\
\text { específicos usados na fase de treino. }\end{array}$ \\
\hline
\end{tabular}




\section{Livro Equivalência}

Entre os artigos que utilizaram o livro equivalência, a maior parte teve como objetivo o desenvolvimento de comportamentos específicos como habilidades acadêmicas (Stanley et al., 2018; Dixon, Belisle, Stanley, Daar, \& Williams, 2016; Dixon, Stanley, et al., 2017, 2017) ou relação entre diferentes sistemas sensoriais (Mullen et al., 2017; McKeel \& Matas, 2017b; Dixon, Belisle, Stanley, et al., 2017).

Os estudos que fizeram uso das relações de equivalência para ensino de habilidades utilizaram diferentes formatos para esse fim. Para o ensino de habilidades de geometria, por exemplo, o estudo de Dixon, Belisle, Stanley, Daar, \& Williams (2016) avaliou o uso de relações de equivalência para ensinar as formas geométricas.

Para a avaliação inicial, foi utilizada a forma estendida da pré-avaliação do PEAK-E; e, para intervenção, um programa desse currículo. No procedimento, foram estabelecidas relações de equivalência entre o nome das formas (A), as propriedades das formas (B) e sua figura (C). Após treino da primeira relação (A-B), ambos os participantes demonstraram simetria (B-A) e transitividade (A-C), sugerindo eficácia desse formato para ensino de habilidades geométricas.

De forma geral, os procedimentos testados envolvendo equivalência foram eficazes para o ensino das habilidades em questão. Em apenas um dos estudos, de McKeel e Matas (2017b), que visava a estabelecer relações entre os sistemas sensoriais gustativo, visual e auditivo, a propriedade de transitividade não foi verificada para um dos três participantes.

Apenas um dos artigos não utilizou procedimentos específicos de equivalência para ensino de relações. Seu objetivo foi investigar se a capacidade de fazer relações de equivalência tinha relação com o comportamento desafiador. Os resultados apontaram que, de fato, em crianças com essa habilidade, era menor a incidência de comportamentos desafiadores (Belisle et al., 2017).

\section{Livro Transformação}

As pesquisas que fizeram uso do livro transformação tiveram como objeto de estudo as relações dêiticas (Belisle, Dixon, Stanley, et al., 2016). Foram pesquisadas as habilidades de tomada de perspectiva eu-você (Barron et al., 2019), identificar eventos privados (emoções) e relações espaciais e temporais sob a forma de "antes-depois" e "aqui-lá". Em um dos estudos (Daar et al., 2015), ainda, foram utilizados procedimentos envolvendo equivalência e transformação para o ensino da habilidade de perguntar e responder perguntas como o que, quem, quando e por quê, também chamadas "Wh- questions".

O estudo de Barron et al. (2019) teve como objetivo o ensino das relações "antes e depois" e "aqui e lá" e foi realizado com duas crianças com autismo. Ambas foram avaliadas com a pré-avaliação do PEAK, mostrando deficit em fazer reversões de quadros dêiticos, assim como identificar quadros dêiticos culturalmente estabelecidos. Foram utilizados dois programas do PEAK-T para treino dessas habilidades por meio de estímulos visuais. Por meio do procedimento de linha de base múltipla cruzando as habilidades, concluiu-se que as duas crianças aprenderam as duas relações (antes/depois e aqui/lá) com transferência de função, assim como aprenderam reversão e transformação para as duas relações previamente ensinadas (Barron et al., 2019).

Os procedimentos envolvendo o livro transformação foram úteis para o ensino das habilidades testadas em todos os estudos selecionados que envolviam esse livro. A única exceção foi no estudo de Daar et al. (2015) envolvendo as "wh-questions", no qual um participante não conseguiu desenvolver a habilidade.

\section{Discussão}

O presente estudo revisou estudos publicados entre 2014-2019 que utilizaram os livros de equivalência e transformação do PEAK. Para as habilidades testadas, os procedimentos envolvendo equivalência e transformação mostraram-se efetivos em todas as pesquisas selecionadas.

Apesar de uma série de estudos estarem sendo conduzidos utilizando também os dois livros iniciais, a proposta foi resgatar o que o PEAK propõe de diferente dos instrumentos já existentes, ou seja, o foco no responder relacional apresentados no PEAK nos livros equivalência de estímulos; e transformação (RFT). 
Em relação às populações estudadas, apesar de quase todos os estudos selecionados, com exceção de um (Dixon, Speelman, et al., 2016), terem como parte da população pessoas com autismo, outros quadros clínicos foram pesquisados, como síndrome de Down e deficiência intelectual, demonstrando aplicabilidade do instrumento para uma variedade de diagnósticos (Dixon et al., 2017).

Nas pesquisas selecionadas, os procedimentos baseados no currículo PEAK foram efetivos para o ensino de uma série de habilidades envolvendo os livros de equivalência e transformação. Tomando como base as relações de equivalência, foi possível o ensino de habilidades bastante distintas entre si, desde habilidades acadêmicas como geometria e geografia até relações entre sistemas sensoriais. De fato, as relações de equivalência já vêm sendo exploradas na literatura para o ensino de diversas habilidades; entre elas, a alfabetização (Souza \& de Rose, 2006). Nesse sentido, o PEAK apresenta-se como uma ferramenta para avaliar essas habilidades de forma padronizada e comparativa, além de propor currículos específicos para possíveis deficits encontrados.

Um questionamento é sobre a decisão do autor de manter equivalência e transformação em livros separados. Uma possível explicação seria a importância do comportamento de relacionar iguais para o aprendizado de outras relações simbólicas e o foco multissensorial do livro, que avalia questões de paladar, olfato, tato, além de audição e visão, que aparecem em toda avaliação.

Outro ponto de destaque é que o referencial teórico do instrumento possibilitou que deficits importantes não focados em avaliações e, muitas vezes, em intervenções habituais, como habilidades de tomada de perspectiva, também chamadas por cognitivistas "teoria da mente", fossem objeto de intervenção de forma sistemática e empírica.

Para analisar tomada de perspectiva, a RFT a enquadra em relações dêiticas com exemplos complexos de responder relacional arbitrariamente aplicado, que, por si, englobam três relações, a saber: interpessoal (EU-VOCÊ), espacial (AQUILÁ) e temporal (AGORA-DEPOIS). Partindo da modelagem dessas respostas relacionais específicas, é possível que habilidades como identificar, interpretar e predizer os pensamentos, os sentimentos e os comportamentos de si próprio ou de outros sejam estudadas e ensinadas (Montoya-Rodrigues et al., 2017).

Os estudos encontrados que abrangeram essas habilidades demonstraram que o instrumento, mais precisamente no livro transformação, pode ser efetivo para desenvolver algumas dessas habilidades. Este achado é relevante tendo em vista a importância desse repertório considerado fundamental na construção de relacionamentos emocionalmente saudáveis consigo e com os outros (Baron-Cohen et al., 1985).

Apesar dos bons resultados no que diz respeito ao uso do PEAK enquanto ferramenta de avaliação e intervenção, o presente estudo de revisão tem limitações importantes. A primeira é que, dos 39 artigos encontrados na busca inicial, apenas dois não fazem parte do grupo de pesquisas que desenvolveu o protocolo. Um passo importante nos estudos envolvendo o PEAK, assegurando resultados de validade interna e externa, seria o uso do programa por grupos de pesquisa diversos. Nesse sentido, Witts (2018) e Reed e Luiselli (2016) indicam a necessidade de mais estudos sobre o tema em diferentes laboratórios.

Outra limitação encontrada diz respeito à idade dos participantes contemplados na presente revisão, sendo que não foram encontrados estudos sobre a utilização do PEAK em intervenção precoce. A maior parte dos estudos é referente a crianças de cinco a nove anos de idade. Além disso, foram encontrados poucos estudos com amostragem de adultos, indicando, novamente, a necessidade de maior abrangência quanto a essa faixa etária em estudos posteriores.

Com base na revisão realizada, o PEAK apresenta-se, então, como uma alternativa de intervenção para o ensino de múltiplas habilidades, possibilitando o ensino de relações simples até relações mais complexas em linguagem, tomada de perspectiva, aprendizagem derivada, e habilidades multissensoriais. Ainda assim, considera-se fundamental a necessidade de mais pesquisas envolvendo o instrumento, para buscar superar as limitações anteriormente citadas.

Apesar das limitações apontadas, o instrumento apresenta uma proposta avançada em relação a outros instrumentos de avaliação direta, como o 
VBMAPP e o ABLLS-R, tanto em relação à ampliação da população em que pode ser aplicado quanto em relação ao repertório-alvo da intervenção, envolvendo operantes relacionais generalizados de diferentes tipos.

\section{Referências}

* Artigos incluídos nesta revisão

Baron-Cohen, S., Leslie, A. M., \& Frith, U. (1985). Do children with autism have a "theory of mind"? Cognition, 21(1), 37-46. https://doi. org/10.1016/0010-0277(85)90022-8

${ }^{\star}$ Barron, B. F., Verkuylen, L., Belisle, J., Paliliunas, D., \& Dixon, M. R. (2019). Teaching "then-later" and "here-there" relations to children with autism: An evaluation of single reversals and transformation of stimulus function. Behavior Analysis in Practice, 12(1), 167-175. http://doi. org/10.1007/s40617-018-0216-1

Belisle, J., Dixon, M. R., \& Stanley, C. R. (2018). The mediating effects of derived relational responding on the relationship between verbal operant development and IQ. Behavior Analysis in Practice, 11(4), 411-416. http://doi. org/10.1007/540617-018-0215-2

* Belisle, J., Dixon, M. R., Stanley, C., Munoz, B., \& Daar, J. H. (2016). Teaching foundational perspective taking skills to children with autism using the PEAK-T curriculum: Single- reversal "I-You" deictic frames. Journal of Applied Behavior Analysis, 49(4), 965-969. http://doi. org/10.1002/jaba.324

Belisle, J., Dixon, M. R., Rowsey, K. E., \& Dixon, M. R. (2016). The use of in-situ behavioral skills training to improve staff implementation of the PEAK relational training system. Journal of Organizational Behavior Management, 36(1), 71-79. https://doi.org/10.1080/01608061.2016. 1152210

* Belisle, J., Stanley, C. R., \& Dixon, M. R. (2017). The relationship between derived mutually entailed relations and the function of challenging behavior in children with autism: Comparing the PEAK-E-PA and the QABF. Journal of Contextual Behavioral Science, 6(3), 298-307. https://doi.org/10.1016/j.jcbs.2017.07.004

Belisle, J., Stanley, C. R., Alholail, A. M., Galliford, M. E., \& Dixon, M. R. (2018). Abstraction of tactile properties by individuals with autism and down syndrome using a picture-based communication system. Journal of Applied Behavior Analysis, 52(2), 467-475. https://doi. org/10.1002/jaba.526

Chomsky, N. (1956). Three models for the description of language. IRE Transactions on Information Theory, 2(3), 113-124. https://doi. org/10.1109/TIT.1956.1056813

* Daar, J. H., Negrelli, S., \& Dixon, M. R. (2015). Derived emergence of $\mathrm{WH}$-question-answers in children with autism. Research in Autism Spectrum Disorders, 19, 59-71. https://doi. org/10.1016/j.rasd.2015.06.004

Dixon, M. R. (2014a). PEAK: Relational Training System: Evidence-based autism assessment and treatment - Direct training module. Carbondale.

Dixon, M. R. (2014b). PEAK: Relational Training System: Evidence-based autism assessment and treatment - Generalization module. Carbondale.

Dixon, M. R. (2015). PEAK: Relational Training System: Evidence-based autism assessment and treatment - Equivalence module. Carbondale.

Dixon, M. R. (2016). PEAK: Relational Training System: Evidence-based autism assessment and treatment - Transformation module. Carbondale.

Dixon, M. R., Belisle, J., McKeel, A., Whiting, S., Speelman, R., Daar, J. H., \& Rowsey, K. (2017). An internal and critical review of the PEAK relational training system for children with autism and related intellectual disabilities: 20142017. The Behavior Analyst, 40(2), 493-521. https://doi.org/10.1007/s40614-017-0119-4

Dixon, M. R., Belisle, J., Munoz, B. E., Stanley, C. R., \& Rowsey, K. E. (2017). Teaching metaphorical extensions of private events through rival-model observation to children with autism. Journal of Applied Behavior, 50(4), 744-749. https://doi. org/10.1002/jaba.418

* Dixon, M. R., Belisle, J., Rowsey, K. E., Speelman, R., Stanley, C., \& Kime, D. (2016). Evaluating emergent naming relations through representational drawing in individuals with developmental disabilities using the PEAK-E curriculum. 
Behavior Analysis: Research and Practice, 17(1), 92-97. https://doi.org/10.1037/bar0000055

* Dixon, M. R., Belisle, J., Stanley, C. R., Daar, J. H., \& Williams, L. A. (2016). Derived equivalence relations of geometry skills in students with autism: An application of the PEAK-E curriculum. The Analysis of Verbal Behavior, 32(1), 3845. https://doi.org/10.1007/s40616-016-0051-9

Dixon, M. R., Belisle, J., Stanley, C., \& Rowsey, K. E. (2018). Student outcomes after 1 year of front-line staff implementation of the PEAK curriculum. Behavioral Interventions, 33(2), 185-195. https://doi.org/10.1002/bin.1516

* Dixon, M. R., Belisle, J., Stanley, C., Munoz, B. E., \& Speelman, R. C. (2017). Establishing derived coordinated symmetrical and transitive gustatory-visual-auditory relations in children with autism and related intellectual disabilities using the PEAK-E curriculum. Journal of Contextual Behavioral Science, 6(1), 91-95. https://doi. org/10.1016/j.jcbs.2016.11.001

Dixon, M. R., Belisle, J., Stanley, C. R., Speelman, R. C., Rowsey, K. E., Kime, D., \& Daar, J. H. (2016). Establishing derived categorical responding in children with disabilities using the PEAK-E curriculum. Journal of Applied Behavior, 50(1), 1-12. https://doi.org/10.1002/jaba.355

Dixon, M. R., Belisle, J., Whiting, S. W., \& Rowsey, K. E. (2014). Normative sample of the PEAK relational training system: Direct training module and subsequent comparisons to individuals with autism. Research in Autism Spectrum Disorders, 8(11), 1597-1606. https://doi. org/10.1016/j.rasd.2014.07.020

Dixon, M. R., Carman, J., Tyler, P. A., Whiting, S. W., Enoch, M. R., \& Daar, J. H. (2014). PEAK relational training system for children with autism and developmental disabilities: Correlations with Peabody Picture Vocabulary Test and assessment reliability. Journal Development Physical Disabilities, 26(5), 603-614. https://doi. org/10.1007/s10882-014-9384-2

* Dixon, M. R., Speelman, R., Rowsey, K. E., \& Belisle, J. (2016). Derived rule-following and transformation of stimulus functions in a children's game: An application of PEAK-E with children with developmental disabilities. Journal of Contextual Behavioral Science,
5(3), 186-192. https://doi.org/10.1016/j. jcbs.2016.05.002

* Dixon, M. R., Stanley, C., Belisle, J., Galliford, M.E., Alholail, A., \& Schmick, A.M. (2017). Establishing derived equivalence relations of basic geography skills in children with autism. Analysis Verbal Behavior, 33(2), 290-295. https://doi.org/10.1007/s40616-017-0084-8

Dixon, M. R., Wiggins, S. H., \& Belisle, J. (2018). The effectiveness of the PEAK relational training system and corresponding chances on the VB-MAPP for young adults with autism. Journal of Applied Behavior Analysis, 51(2), 321-344. https://doi.org/10.1002/jaba.448

Dymond, S., \& Rehfeldt, R. A. (2000). Understanding complex behavior: The transformation of stimulus functions. The Behavior Analyst, 23(2), 239-254. https://doi. org/10.1007/BF03392013

Dymond, S., May, R. J., Munnelly, A., \& Hoon, A. E. (2010). Evaluating the evidence base for relational frame theory: A citation analysis. Behavior Analyst, 33(1), 97-117. https://doi.org/10.1007/ BF03392206

Hayes, S. C., Barnes-Holmes, D., \& Roche, B. (2001). Relational frame theory: A post-Skinnerian account of human language and cognition. Plenum Press.

Malkin A., Dixon, M. R., Speelman, R. C., \& Luke, N. (2016). Evaluating the relationships between the PEAK relational training system - Direct training modules, assessment of basic language and learning skills - Revised, and the Vineland adaptative behavior scales - II. Journal of Developmental and Physical Disabilities, 29(2), 341-351. https://doi. org/10.1007/s10882-016-9527-8

Matsumoto, M. S. (2014). A generalização de repertórios de mando após o ensino a crianças com autismo: Uma revisão de artigos de 2009 a 2014 [Dissertação de mestrado, Núcleo Paradigma de Análise do Comportamento, São Paulo, SP, Brasil]. https://www.nexoic. com.br/wp-content/uploads/2019/07/A- generaliza \% C $3 \%$ A 7 \% C $3 \%$ A 3 o-de-repert\%C3\%B3rios-de-mando-ap\%C3\%B3s-o-ensino-a-crian\% $\mathrm{C} 3 \% \mathrm{~A} 7 \mathrm{as}$-com-autismo_ uma-revis\%C3\%A3o-de-artigos-de-2009-a-2014.pdf 
* McKeel, A., \& Matas, J. (2017b). Utilizing PEAK relational training system to teach visual, gustatory, and auditory relations to adults with developmental disabilities. Behavior Analysis in Practice, 10(3), 252-260. https://doi. org/10.1007/s40617-017-0194-8

McKeel, A. N., Rowsey, K. E., Belisle, J., Dixon, M. R., \& Szekely, S. (2015). Teaching complex verbal operants using the PEAK relational training system. Behavior Analysis in Practice, 8(2), 241-244. https://doi.org/10.1007/s40617-0150067-y

Mcllvane, W. (2003). A stimulus in need of a response: A review of relational frame theory: A post Skinnerian account of human language and cognition. The Analysis of Verbal Behavior, 19(1), 29-37. https://doi.org/10.1007/ BF0339298

Montoya-Rodriguez, M. M, Molina, F. J., \& McHugh, L. (2017). A review of relational frame theory research into deictic relational responding. The Psychological Record, 67(4), 569-579. https://doi.org/10.1007/s40732-0160216-x

* Mullen, S., Dixon, M. R., Belisle, J., \& Stanley, C. (2017). Establishing auditory-tactile-visual equivalence classes in children with autism and developmental delays. Analysis Verbal Behavior, 33(2), 283-289. https://doi.org/10.1007/ s40616-017-0092-8

Perez, W. F., Nico, Y. C., Kovac, R., Fidalgo, A. P., \&Leonardi, J. L. (2013). Introdução à teoria das molduras relacionais (relational frame theory): Principais conceitos, achados experimentais e possibilidades de aplicação. Perspectivas em Análise do Comportamento, 4(1), 33-51. http:// pepsic.bvsalud.org/scielo.php?script=sci_arttex t\&pid=S2177-35482013000100005

Reed, D., \& Luiselli, J. (2016). Promoting the emergence of advanced knowledge: A review of PEAK relational training system: direct training module by Mark R. Dixon. Journal of Applied Behavior Analysis, 49, 205-211. https://doi. org/10.1002/jaba.281

* Schmick, A. M., Stanley, C.R., \& Dixon, M. R. (2018). Teaching children with autism to identify private events of other in context. Behavior Analysis in Practice, 11(4), 400-405. doi: 10.1007/s40617-018-0214-3

Sidman, M. (1971). Reading and auditory-visual equivalences. Journal of Speech, Language, and Hearing Research, 14(1), 5-13. https://doi. org/10.1044/jshr.1401.05

Sidman, M., \& Tailby, W. (1982). Conditional discrimination vs. matching to sample: An expansion of the testing paradigm. Journal of the Experimental Analysis of Behavior, 37(1), 5-22. https://doi.org/10.1901/jeab.1982.37-5

Skinner, B. F. (1978). O comportamento verbal (M. P. Villalobos, Trad.). São Paulo: Cultrix/Edusp. (Trabalho original publicado em 1957)

Souza, D.G., \& de Rose, J. (2006). Desenvolvendo programas individualizados para o ensino de leitura. Revista Latina de Análisis de Comportamiento, 14(1), 77-98. http://pepsic. bvsalud.org/scielo.php?script=sci_arttext\&pid $=$ S0188-81452006000100004

* Stanley R., Belisle, J., \& Dixon, M. R. (2018). Equivalence-based instruction of academic skills: Application to adolescents with autism. Journal of Applied Behavior Analysis, 51(2), 352-359. https://doi.org/10.1002/jaba.446

Witts, B. N. (2018). An external review of the conclusions regarding the PEAK direct training module. Journal of Applied Behavior Analysis, 51(3), 719-737. https://doi.org/10.1002/ jaba.491

\section{Informações do Artigo}

Histórico do artigo:

Submetido em: 24/11/2020

Primeira decisão editorial: 18/04/2021

Aceito em: 02/08/2021

Editor: William F. Perez 